Originales

\title{
Resultados de una encuesta nacional sobre hábito transfusional en unidades de cuidados intensivos
}

\author{
MANUEL QUINTANA DÍAZa , MARCELINO SÁNCHEZ CASADO ${ }^{b}$, SANTIAGO RAMÓN LEAL NOVALc \\ ABELARDO GARCÍA DE LORENZO Y MATEOS ${ }^{a} Y$ GRUPO DE TRABAJO DE HEMODERIVADOS \\ Y ALTERNATIVAS TRANSFUSIONALES

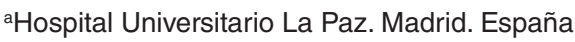 \\ bHospital Virgen de la Salud. Toledo. España. \\ 'Hospital Universitario Virgen del Rocío. Sevilla. España.
}

Introducción. Los hemoderivados son productos clínicos de uso habitual que presentan unas características de escasez, relevancia clínica y de efectos secundarios concomitantes que obligan a conocer y evaluar su utilización con rigurosidad.

Objetivo. Analizar actitudes, conocimientos y conductas sobre la política transfusional de las diferentes unidades de cuidados intensivos (UCI) españolas.

Diseño. Encuesta mediante correo (electrónico y tradicional) en las unidades de cuidados intensivos.

Periodo. Planificación durante 2004 y realización durante todo 2005.

Ambito. UCl españolas.

Pacientes y método. Cuestionario de 27 preguntas.

Resultados. La mayoría son UCI de tamaño medio (10-20 camas), con actividad predominantemente médica. El adjunto, solo o con el residente, suele decidir la transfusión según su experiencia, aunque con tendencia a seguir las recomendaciones científicas. No suele haber comités transfusionales. Cuando se transfunden concentrados de hematíes, suelen ser entre 2 y 4 unidades. La cifra de hemoglobina es orientativa, aunque la decisión es clara si es $<7$ g/dl $(10 \mathrm{~g} / \mathrm{dl}$ si hay cardiopatía). No se suele emplear alternativas farmacológicas a las transfusiones por motivos de escasa evidencia y precio. En el $50 \%$ de los casos

Correspondencia: Dr. M. Quintana Díaz.

Servicio de Urgencias General. Hospital Universitario La Paz

Paseo de la Castellana, 261. 28046 Madrid. España.

Correo electrónico: mdiazq.hulp@salud.madrd.org

Manuscrito aceptado el 28-5-2008. se considera cierta la relación entre transfusión y aumento de mortalidad.

Conclusiones. Debemos reflexionar sobre la transfusión y evitar la sobreutilización. Para ello se necesitan protocolos educacionales y conferencias de consensos que establezcan recomendaciones sobre el uso de los hemoderivados y sus alternativas farmacológicas.

PALABRA CLAVE: Transfusión sanguínea. Práctica transfusional. Encuesta. Paciente crítico.

\section{RESULTS OF A NATIONAL SURVEY ON TRANSFUSION HABIT IN INTENSIVE CARE UNITS}

Introduction. Blood derivatives are clinical products that are currently used, for which their lack of availability, clinical relevance and presence of associated side effect that make it necessary to known and evaluate their utility rigorously are characteristic.

Objective. To analyze knowledge of attitudes, knowledge and behavior on transfusional policy in the different Spanish Intensive Care Units (ICU).

Design. A mail-based survey (electronic and conventional) in the ICUs.

Duration. The study was planned in 2005 and conducted during the year 2006.

Setting. Spanish ICUs.

Patients and Method. A 27-question questionnaire.

Results. Most are middle-sized ICUs (10-20 beds), with predominantly medical activities. The staff member, alone or with the resident, generally decides the transfusion based on his/her experience, although with a tendency to follow 
the scientific guidelines. Generally, there is no transfusional committee. When red blood packs are transfused, generally between 2 to 4 units are used. The hemoglobin value is orientative, although the decision is clear if $<7 \mathrm{~g} / \mathrm{dl}(10 \mathrm{~g} / \mathrm{dl}$ if there is heart disease). Drug alternatives to transfusion are not generally used due to lack of evidence and price. In $50 \%$ of cases, the association between transfusion and increase in mortality is considered to be certain.

Conclusions: Strong consideration must be made about transfusion and its over use should be avoided. For this purpose, educational guidelines and consensus meetings are necessary to establish recommendations on the use of blood products and their pharmacological alternatives.

KEY WORDS: Blood transfusion. Blood transfusion practices. Survey. Critically ill.

\section{INTRODUCCIÓN}

«Lo que sabemos, lo sabemos juntos»

Hans Sigaard Jensen

Los pacientes críticos reciben con frecuencia una transfusión sanguínea durante su estancia en las unidades de cuidados intensivos (UCI) ${ }^{1-4}$. La escasez de los hemoderivados y los efectos secundarios relacionados con su relevancia clínica han determinado la realización de conferencias de consenso y guías clínicas que orientan su utilización de una manera más rigurosa ${ }^{3,5-7}$.

El uso de cuestionarios, como instrumento de medida, es una forma cada vez más frecuente de acercarse a la realidad clínica, determinar las características de los pacientes o evaluar el cuidado sanitario. Esto se ve apoyado por su bajo coste y porque se realizan de forma relativamente rápida e «incruenta».

A pesar de las limitaciones metodológicas de este tipo de estudios, es un método que ayuda a dar un panorama general de la práctica clínica, por lo que ha sido utilizado con frecuencia en diferentes países con el objetivo específico de describir la utilización de hemoderivados en diferentes contextos clínicos; su empleo en las UCI ha sido escaso ${ }^{8-19}$.

El Grupo de Hemoderivados de la SEMICYUC planteó la realización de una encuesta nacional con el fin de conocer la realidad del hábito transfusional en las UCI españolas, mediante el análisis de las actitudes, los conocimientos y las conductas sobre la política transfusional de estas unidades.

\section{MATERIAL Y MÉTODO}

Durante el año 2004 el Grupo de Hemoderivados de la SEMICYUC planificó la encuesta, y contactó con las UCI españolas, invitándolas a participar. Se seleccionaron 84 UCI, representativas del panorama nacional. Durante todo el año 2005 se inició el reclutamiento, se recogieron las encuestas completadas y se presentaron los datos definitivos.

La encuesta constaba de 27 preguntas, con opciones múltiples y cerradas. En el cuestionario se plan- tean varios casos clínicos para valorar la respuesta ante determinados contextos de actuación.

Se contactó con hospitales públicos y privados, para ello se utilizó el correo electrónico y el convencional. Dentro de cada UCI, uno o dos intensivistas respondían al cuestionario.

Se ha intentado responder a los siguientes aspectos de interés: características generales (distribución geográfica, tamaño y actividad de las diferentes UCI), quién transfunde (quién toma la decisión, con qué criterios y si hay protocolos y comités de transfusiones), cuándo transfundimos (porcentaje de pacientes, requerimientos transfusionales), qué transfundimos (hematíes, plaquetas y plasma), por qué transfundimos (por la cifra de hematíes, en contextos de ausencia o presencia de sangrado) y alternativas a la transfusión empleadas (existencia de recuperadores, uso de eritropoyetina, aprotinina, hierro y factor VII activado). El cuestionario completo se incluye en el anexo 1 .

Los datos son todos categóricos, y se expresan como número absoluto (porcentaje). Se han realizado comparaciones entre diferentes grupos (según tamaño de la UCI, etc.) con la prueba de la $\chi^{2}$. Si no se cumplían las condiciones de aplicación, se utilizó el test exacto de Fisher. Se considera significativa una $\mathrm{p}$ $<0,05$.

\section{RESULTADOS}

Se han analizado 84 encuestas procedentes de UCI; contestaron la encuesta la totalidad de las unidades elegidas. La distribución geográfica se muestra en la figura 1. Un representante conocido de cada unidad fue contactado y realizó la encuesta en solitario o con ayuda de alguno de sus compañeros. Se recibieron todas las encuestas completadas de forma plena en la mayoría de las preguntas. La tasa de respuestas contestadas en las encuestas recibidas oscila entre el 93 y el 100\% (grado de respuesta adecuado).

Se realizaron preguntas relacionadas con las características de las UCI estudiadas (tabla 1), las características de las decisiones de transfusión (tabla 2), la valoración de la cantidad transfundida de hemoderivados (tabla 3 ) y si hay alternativas a la transfusión sanguínea (tabla 4).

También se realizaron una serie de preguntas específicas y casos clínicos que pudieran representar la actitud tomada en las diferentes UCI ante ejemplos prácticos. Las respuestas a estas cuestiones figuran en la tabla 5 .

Se ha conseguido una distribución homogénea en cuanto al nivel del hospital. La mayoría son UCI de tamaño medio (de 10-20 camas), con actividad predominantemente médica. El grupo de politraumatizados es el que con más frecuencia aparece en las unidades como un grupo especial de grandes requerimientos transfusionales (el $81 \%$ de las unidades). Son pocas las unidades en donde se realiza trasplante.

El adjunto, solo o con el residente, suele decidir la transfusión según su experiencia y las recomendacio- 


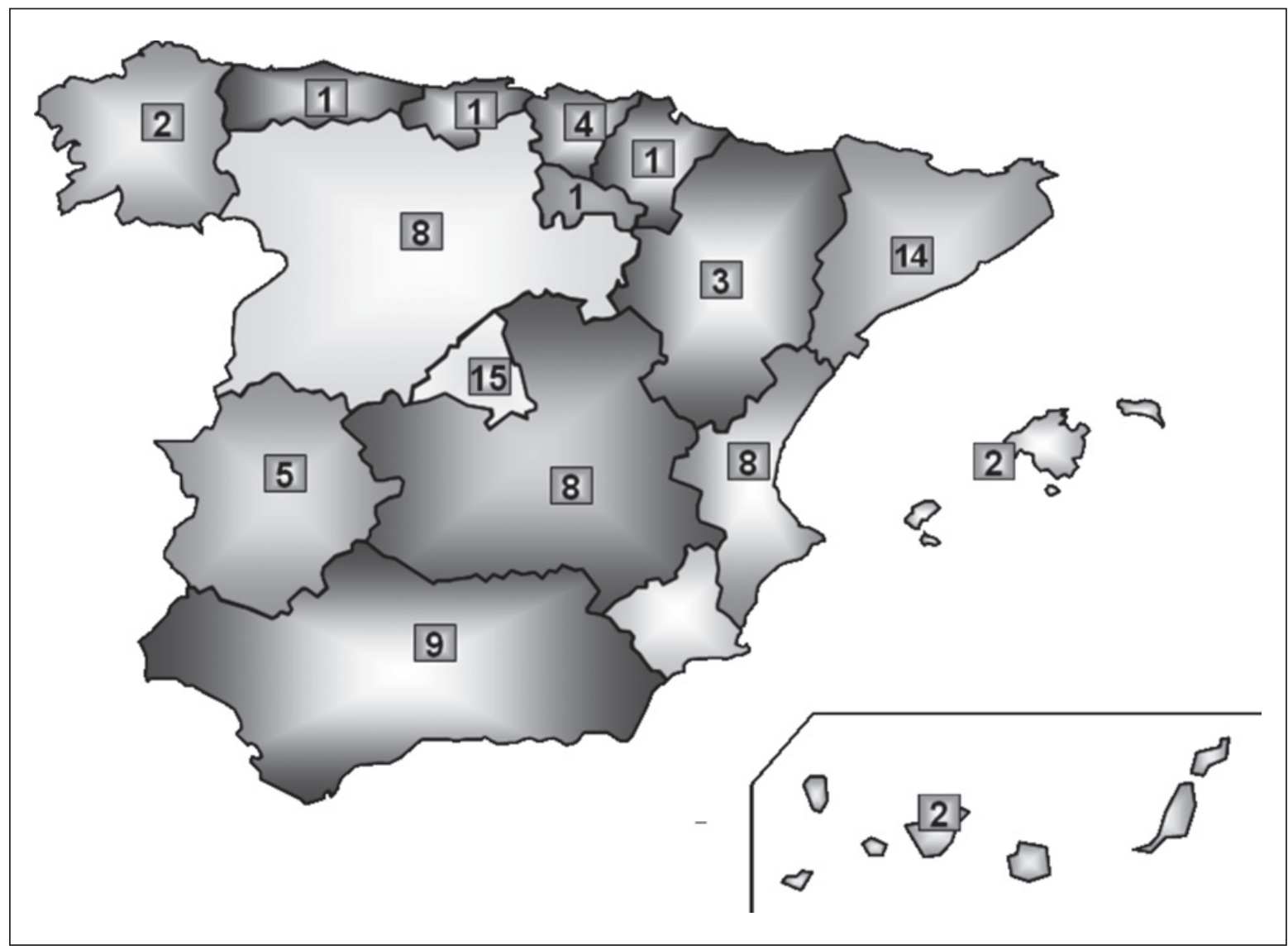

Figura 1. Distribución de las unidades que han participado en la encuesta.

H. Ntra. Sra. del Prado (Dr. M. Quintana); H. Vega Baja (Dra. M.T. Blanco/Dra. D. Olivares); H. de Castelló (Dr. R. Abizanda); F.H. Manacor (Dr. R. Pitarch); H. Juan Canalejo (Dra. R. Arrojo); H. de Pontevedra (Dr. E. Alemparte); C.H. San Millan (Dr. A. Moreno); H. Central de Asturias (Dr. G. Rey); H. Virgen del Camino (Dr. J. Izura); H. Virgen de la Salud (Dr. M. Rodriguez); C.H. Albacete (Dra. G. Vega); C.H. Mancha-Centro (Dr. R. Blancas); C.H. Ciudad Real (Dra. C. Martin); H. Virgen de la Luz (Dr. J. Añon); H. de Guadalajara (Dr. C. Almendariz); H. Provincial de Toledo (Dr. D. Cabestrero); H. Río Carrión (Dr. J. Lopez. Messa); H. Nuestra Sra. de Sonsoles (Dr. A. Isusi); C.H. Clínico Salamanca (Dr. J.C. Ballesteros); H. Virgen de la Concha (Dra. T.L. Alvarez); H. El Bierzo (Dra. C. Matinez); H. La Ribera (Dra. I. Rueda); H. de Cruces (Dr. J. Zaldumbide); H. Santiago Apostol (Dr. J. Maynar); Clínica Quirón (Dra. I. Valduvieco); C.H. Donostia (Dr. F. Lavado); H. General Yagüe (Dr. A. Zabalegui); H. 12 de Octubre (Dr. J.C. Montejo); H. Puerta de Hierro (Dr. C. Chamorro); Fundación Jiménez Díaz (Dra. A. Gamo); F.H. Alcorcón (Dra. S. Temprano); H. La Paz (Dr. A. García de Lorenzo); H. Central de la Defensa (Dr. Hernández Abadia); H. Gregorio Marañón (Dr. E. Palencia); H. Severo Ochoa (Dr. F. Del Nogal); H. de Getafe (Dr. M.A. De la Cal); Clínica Moncloa (Dr. V. Gómez Tello); H. Ramón y Cajal (Dra. C. Pintado); H. Clínico San Carlos (Dr. A. Blesa); C. H. de Móstoles (Dra. R. Asensio); H. Príncipes de Asturias (Dra. M.E. Fuente); H. Verge de Toro (Dr. R. Fernández Cid); H. Virgen de las Nieves (Dr. E. Fernández Mondejar); H. San Juan de Dios (Dra. I. Jara); H. Virgen del Rocío (Dra. M.D. Rincón); H. Virgen de la Victoria (Dra. V. De la Torre); Clínica Casa de la Salud (Dr. J. Ruiz); H. La Fe (Dr. S. Borras); H. de Vinarós (Dr. F. Barrachina); H. de Xagunto (Dra. E. Gómez); H. Lluís Alcanyís (Dra. D. García); H. Princesa Sofía (Dr. R. Guerrero); H. de Soria (Dr. P. Medina); H. de Segovia (Dra. M.J. Lopez Cambra); H. Río Hortega (Dr. J. Blanco); H. Obispo Polanco (Dr. I. Lorda); H. Lozano Blesa (Dra. P. Luque); H. Virgen del Puerto (Dra. M.J. Chavero); C.H. de Cáceres (Dra. M. Montans); H. de Mérida (Dr. F. Andrade); H. de Don Benito (Dr. M. Cidoncha); H. Marqués de Valdecilla (Dr. J.C. Rodriguez); H. German Trias i Pujol (Dr. P. Torrabadella); H. del Mar (Dr. F. Solsona); H. Dr. Trueta (Dr. A. Alvarez Dra. N. Masnou); H. Barcelona-SCIAS (Dr. L. Cabré); Mutua de Terrassa (Dra. M.T. Jurado); H. Vall d'Hebron (Dr. X. Nuvials); H. Insular (Dr. M. Sánchez Palacios); H. de Fuerteventura (Dra. C. Vaquero); H. de Bellvitge (Dr. J. Díaz); H. Carlos Haya (Dr. G. Jimenez); H. Arnau i Vilanova (Dra. M. Badia); F.H. Fuenlabrada (Dr. M. Borrallo); H. Clínico San Cecilio (Dr. S. Schaffino); H. de Granollers (Dr. P. Garro); H. Verge de la Cinta (Dr. G. Masdeu); H. Vall d'Hebron (Trauma) (Dr. F.G. Rey); H. Vall d'Hebron (Cardíaca) (Dr. J. Roma); H. Parc Taulí (Dr. R. Ferrer); H. San Juan (Dr. C. Antón); Clínica Delfos Barna (Dr. F. Fernández); Clínica Salus Benalmádena (Dr. G. Jimenez); H. Infanta Cristina (Dr. D. Perez Civantos).

nes de consenso. No suele haber protocolos en las UCI ni comités transfusionales en el hospital. Cuando hay comités, éstos no suelen generar normas o tienen escasa relevancia.

El porcentaje de transfusión referido es alto (en el $42 \%$ de las unidades entre el 20 y el $40 \%$ ). Raramente se transfunde un concentrado de hematíes; la media de concentrados transfundidos es de 2-4 unidades. La transfusión de plaquetas ocurre en menos del $5 \%$ de los pacientes y el de plasma, en el 5-15\% de los pacientes.

La cifra de hemoglobina es orientativa, aunque la decisión es clara si es $<7 \mathrm{~g} / \mathrm{dl}$ ( $10 \mathrm{~g} / \mathrm{dl}$ si hay cardiopatía). No se suele emplear alternativas farmacológi- 


\section{ANEXO 1. Cuestionario completo}

1. El número de camas de su UCI es:

a. $<10$

b. $10-20$

c. $21-30$

d. $>30$

2. La actividad de su UCI es:

a. Médica exclusivamente

b. Quirúrgica exclusivamente.

c. Médico-quirúrgica $(50 \%)$

d. Médico-quirúrgica (predominante médica, más del $60 \%$ de camas)

e. Médico-quirúrgica (predominantemente quirúrgica más del 60\% de camas)

3. En su unidad se trata a pacientes con altos requerimientos transfusionales:

a. Trasplante cardíaco

b. Trasplante hepático

c. Cirugía cardíaca

d. Politraumatizados

4. ¿El comité de transfusiones sanguíneas de su hospital genera normas, conocidas por usted, respecto al uso de sangre en UCI?

a. Sí. El comité está involucrado en las transfusiones del paciente crítico y sus alternativas. Sus normas son seguidas por los intensivistas

b. Sí. El comité está involucrado en las transfusiones del paciente crítico y sus alternativas. Sus normas tienen escasa/nula relevancia para los intensivistas

c. El comité de mi hospital no genera normas que afecten al consumo de sangre del paciente crítico

d. En mi hospital no hay comité de transfusiones

5. En su unidad las transfusiones de sangre se basan predominantemente:

a. En un protocolo elaborado y consensuado por su servicio

b. No hay protocolo, pero se siguen recomendaciones de guías o conferencias de consenso, conocidas por los médicos

c. En la experiencia y el criterio del médico responsable del paciente

6. La decisión de transfundir la toma habitualmente:

a. Médico adjunto responsable del paciente

b. Médico residente

c. Indistintamente

7. ¿Se transfunde una sola unidad de concentrado de hematíes?

a. Frecuentemente

b. Raramente

c. Nunca

8. El porcentaje de pacientes transfundidos con cualquier componente sanguíneo (hematíes/plasma y/o plaquetas) es:

a. $<20 \%$

b. $20-40 \%$

c. $>40 \%$

9. Los pacientes transfundidos electivamente ( $\sin$ hemorragia) reciben una media de concentrado de hematíes de:

a. $<2$

b. $2-4$

c. $>4$

10. El porcentaje de pacientes transfundidos con plasma en su unidad es de:

a. $<5 \%$

b. $5-15 \%$

c. $15-25 \%$

d. $>25 \%$

11. El porcentaje de pacientes transfundidos con plaquetas en su unidad es de:

a. $<5 \%$

b. $5-15 \%$

c. $15-25 \%$

d. $>25 \%$

12. Generalmente, usted trata la anemia del paciente crítico con:

a. Sangre total

b. Concentrado de hematíes al $55 \%$

c. Concentrado de hematíes al $80 \%$

13. Si un paciente sin hemorragia ingresado en su UCI requiere cirugía electiva, usted le transfunde hasta que la hemoglobina prequirúrgica $>100 \mathrm{~g} / 1$

a. Siempre

b. Nunca

c. Siempre que tenga antecedentes de cardiopatía

14. Paciente de 15 años ingresado en UCI en el postoperatorio de cirugía cardíaca (cierre de comunicación interauricular). No sangra ni tiene problemas cardiorrespiratorios. ¿Con qué valor de hemoglobina indicaría usted una transfusión?

a. $70 \mathrm{~g} / 1$

b. $90 \mathrm{~g} / 1$

c. $100 \mathrm{~g} / 1$

d. No indicaría transfusión, independientemente de la hemoglobina 


\section{ANEXO 1. Cuestionario completo (continuación)}

15. Paciente de 70 años ingresado en UCI en el postoperatorio de reparación de aneurisma abdominal roto. No sangra ni tiene problemas cardiorrespiratorios. ¿Con qué cifra de hemoglobina indicaría usted una transfusión?

a. $70 \mathrm{~g} / 1$

b. $90 \mathrm{~g} / 1$

c. $100 \mathrm{~g} / 1$

d. No indicaría transfusión, independientemente de la hemoglobina

16. Paciente de 23 años ingresado en UCI por politraumatismo $(5 \mathrm{~h}$ después del accidente). No parece sangrar y está sometido a ventilación mecánica con concentración de oxígeno inferior al 40\%. Su presión arterial sistólica es de 120 mmHg. ¿Con qué cifra de hemoglobina indicaría usted una transfusión?

a. $70 \mathrm{~g} / 1$

b. $90 \mathrm{~g} / 1$

c. $100 \mathrm{~g} / 1$

d. No indicaría transfusión, independientemente de la hemoglobina

17. Paciente de 40 años ingresado en UCI en el postoperatorio de trasplante hepático (realizado $4 \mathrm{~h}$ antes). Sangrado escaso por los drenajes (menos de $60 \mathrm{ml} / \mathrm{h}$ ) y no tiene problemas cardiorrespiratorios. ¿Con qué cifra de hemoglobina indicaría usted una transfusión?

a. $70 \mathrm{~g} / 1$

b. $90 \mathrm{~g} / 1$

c. $100 \mathrm{~g} / 1$

d. No indicaría transfusión, independientemente de la hemoglobina

18. En un paciente con sangrado posquirúrgico, ¿en qué circunstancias transfundiría concentrado de plaquetas?

a. Plaquetas posquirúrgicas $<30 / 10^{9}$

b. Plaquetas posquirúrgicas $<100 / 10^{9}$

c. Se le han transfundido $>10$ unidades de concentrado de hematíes

d. Tomaba antiagregantes plaquetarios en la semana previa a la cirugía

19. Además de la hemoglobina pretransfusional, ¿tiene usted en cuenta otros factores antes de transfundir?

a. Sí. La hemoglobina es sólo un factor más en la decisión de transfundir

b. No. La cifra de hemoglobina es el marcador fundamental por el que decido la transfusión

20. En pacientes sin hemorragia ni riesgo de sangrado que amenace la vida, y que no vayan a requerir cirugía urgente, la transfusión de plasma y/o plaquetas es:

a. Se prescribe acorde al estudio de coagulación

b. Se prescribe sólo en situaciones muy especiales y si no hay alternativas farmacológicas

c. Casi nunca/nunca se prescribe

21. En su UCI, se usa algún aparato para recuperar sangre en el periodo postoperatorio y reinfundirla al paciente?

a. Sí

b. No

c. No lo sé

22. ¿Se usa en su UCI eritropoyetina para prevenir o tratar la anemia del paciente crítico? (fuera de la indicación de insuficiencia renal y/o neoplasia)

a. Nunca

b. Esporádicamente $(<20 \%$ de los pacientes anémicos $)$

c. Frecuentemente (20-80\% de los pacientes anémicos)

d. Siempre

23. ¿Se usa en su UCI aprotinina en cirugía cardíaca y/o trasplante?

a. Nunca

b. Esporádicamente $(<20 \%$ de los pacientes de cirugía cardíaca)

c. Frecuentemente (20-80\% de los pacientes de cirugía cardíaca)

d. Siempre

24. ¿Se usa en su UCI factor VII recombinante en pacientes con hemorragia incontrolable (cirugía cardíaca, trasplante,

politraumatismo, gran quemado)?

a. Nunca

b. Anecdóticamente (menos de 5 casos al año)

c. Esporádicamente (más de 5 casos al año)

25. El uso en su UCI de alternativas farmacológicas a las transfusiones de sangre está limitado por:

a. No creo que existan alternativas farmacológicas eficaces a las transfusiones de sangre

b. La evidencia de su eficacia es escasa

c. Economía (fármacos caros)

d. En mi medio no hay limitaciones y pueden ser usados regularmente

26. ¿Cree usted en las aseveraciones de los recientes estudios multicéntricos europeo (ABC Study; Vincent et al. JAMA. 2002;288:1499-507) y americano (CRIT Study; Corwin et al. Crit Care Med. 2004;32:39-52) que asocian las transfusiones sanguíneas con incremento de la morbimortalidad, en el paciente crítico?

a. Sí. Creo que hay una clara asociación entre transfusión y morbimortalidad. Las transfusiones pueden ser causantes de incremento de la morbimortalidad

b. Sí. Creo que hay una clara asociación entre transfusión y morbimortalidad. Esta asociación, sin embargo, es circunstancial. Las transfusiones no han demostrado un incremento de la morbimortalidad

c. No conozco los estudios referidos 
TABLA 1. Características de las unidades de cuidados intensivos (UCI) estudiadas

\begin{tabular}{|lc|}
\hline \multicolumn{1}{|c|}{ Variable } & $\mathrm{n}(\%)$ \\
\hline Nivel de hospital & \\
I & $20(28,6 \%)$ \\
II & $26(37,1 \%)$ \\
III & $24(34,3 \%)$ \\
Número de camas UCI & $17(20,2 \%)$ \\
< 10 camas & $51(60,7 \%)$ \\
10-20 & $9(10,7 \%)$ \\
21-30 & $7(8,3 \%)$ \\
> 30 & \\
Actividad UCI & $14(16,7 \%)$ \\
Médico-quirúrgica (predominantemente & $27(32,1 \%)$ \\
$\quad$ quirúrgica > 60\%) & \\
Médico-quirúrgica (50\%) & $43(51,2 \%)$ \\
Médico-quirúrgica (predominantemente & \\
$\quad$ médica > 60\%) & $55(65,5 \%)$ \\
Altos requerimientos transfusionales & $7(8,3 \%)$ \\
Politraumatizados & $1(1,2 \%)$ \\
Cirugía cardíaca & $2(2,6 \%)$ \\
Trasplante cardíaco & $6(7,1 \%)$ \\
Trasplante hepático & $3(3,6 \%)$ \\
Trasplante cardíaco y hepático & $10(11,9 \%)$ \\
Politraumatizados y cirugía cardíaca & \\
Todos & \\
\hline
\end{tabular}

TABLA 2. Características de las decisiones de transfusión

\begin{tabular}{|l|c|}
\hline \multicolumn{1}{|c|}{ Variable } & $\mathrm{n}(\%)$ \\
\hline Decisión transfusión & \\
Médico adjunto & $53(63,1 \%)$ \\
Médico residente & $1(1,2 \%)$ \\
Indistintamente & $30(35,7 \%)$ \\
Base de la transfusión & $36(42,9 \%)$ \\
Experiencia y criterio médico & $43(51,2 \%)$ \\
Recomendaciones de consenso conocidas & $4(4,8 \%)$ \\
Protocolo elaborado y consensuado & $37(44 \%)$ \\
Comité de transfusiones & $21(25 \%)$ \\
No hay & $15(17,9 \%)$ \\
No genera normas de consumo & $10(11,9 \%)$ \\
Involucrado; normas con escasa relevancia & \\
Involucrado; normas seguidas &
\end{tabular}

cas a las transfusiones por motivos de escasa evidencia y precio. La utilización de recuperadores de sangre y eritropoyetina y factor VIIa es baja. En el $50 \%$ de los casos se considera que hay relación entre transfusión y aumento de mortalidad en los pacientes críticos.

Hemos intentado determinar si hay diferencias en la forma de responder según el tamaño de la UCI o su actividad, pero no hay diferencias significativas entre grupos.

\section{DISCUSIÓN}

El uso de cuestionarios con frecuencia plantea problemas, desde su elección hasta las dificultades metodológicas para asegurar la validez de los resultados. Esto no es óbice para intentar realizar medidas obje-
TABLA 3. Valoración de la cantidad transfundida de hemoderivados

\begin{tabular}{|lc|}
\hline \multicolumn{1}{|c|}{ Variable } & $\mathrm{n}(\%)$ \\
\hline Transfusión cualquier componente & \\
$<20 \%$ & $39(46,9 \%)$ \\
$20-40 \%$ & $35(42,2 \%)$ \\
$>40 \%$ & $9(10,8 \%)$ \\
Transfusión de 1 concentrado de hematíes & $12(14,3 \%)$ \\
Frecuentemente & $43(51,2 \%)$ \\
Raramente & $29(34,5 \%)$ \\
Nunca & \\
Media de concentrados de hematíes & \\
en transfusión electiva & $15(17,9 \%)$ \\
$<2$ & $69(82,1 \%)$ \\
$2-4$ & \\
Porcentaje de pacientes transfundidos & \\
con plasma & $33(40,2 \%)$ \\
$<5 \%$ & $40(48,8 \%)$ \\
$5-15 \%$ & $9(11 \%)$ \\
$15-25 \%$ & \\
Porcentaje de pacientes transfundidos & \\
con plaquetas & $51(62,2 \%)$ \\
$<5 \%$ & $24(29,3 \%)$ \\
$5-15 \%$ & $7(8,5 \%)$ \\
$15-25 \%$ &
\end{tabular}

TABLA 4. Alternativas a la transfusión sanguínea

\begin{tabular}{|c|c|}
\hline Variable & $\mathrm{n}(\%)$ \\
\hline \multicolumn{2}{|l|}{$\begin{array}{l}\text { Utilización de recuperadores de sangre } \\
\text { en postoperatorio y reinfusión }\end{array}$} \\
\hline Sí & $8(9,5 \%)$ \\
\hline No & $76(90,5 \%)$ \\
\hline \multicolumn{2}{|l|}{$\begin{array}{l}\text { Eritropoyetina para prevenir o tratar anemia } \\
\text { en paciente crítico }\end{array}$} \\
\hline Nunca & $60(71,4 \%)$ \\
\hline Esporádicamente $(<20 \%$ pacientes anémicos $)$ & $24(28,6 \%)$ \\
\hline \multicolumn{2}{|c|}{ Aprotinina en cirugía cardíaca y/o trasplante } \\
\hline No hay esa patología & $31(36,9 \%)$ \\
\hline Nunca & $31(36,9 \%)$ \\
\hline Esporádicamente $(<20 \%)$ & $14(16,7 \%)$ \\
\hline Frecuentemente $(20-80 \%)$ & $5(6 \%)$ \\
\hline Siempre & $3(3,6 \%)$ \\
\hline \multicolumn{2}{|l|}{$\begin{array}{l}\text { Factor VII recombinante en hemorragia } \\
\text { incontrolable }\end{array}$} \\
\hline Nunca & $27(32,1 \%)$ \\
\hline Anecdóticamente $(<5$ casos/año $)$ & $46(54,8 \%)$ \\
\hline Esporádicamente (> 5 casos/año) & $11(13,1 \%)$ \\
\hline \multicolumn{2}{|l|}{$\begin{array}{l}\text { Limitación de alternativas farmacológicas } \\
\text { a transfusiones }\end{array}$} \\
\hline Economía (fármacos caros) & $26(31 \%)$ \\
\hline Evidencia de su eficacia & $19(22,6 \%)$ \\
\hline Sin limitaciones y uso regular & $16(19 \%)$ \\
\hline No creo en estas alternativas & $14(16,7 \%)$ \\
\hline Evidencia escasa y fármacos caros & $9(10 \%)$ \\
\hline
\end{tabular}

tivas de muestras representativas que, aplicadas de forma sistemática, permitan comparar los resultados a lo largo del tiempo. Esto se acompaña por la multitud de guías y consensos realizados en este campo durante todos estos años.

Esto llevó al grupo de hemoderivados de la SEMICYUC a realizar una encuesta que permita te- 
TABLA 5. Preguntas clínicas y casos específicos planteados en el cuestionario

\begin{tabular}{|c|c|}
\hline Número de pregunta & Enunciados (\% respuestas) \\
\hline \multirow[t]{2}{*}{12} & Concentrados de hematíes al $80 \%(59,5 \%)$ \\
\hline & Concentrados de hematíes al $55 \%(35,7 \%)$ \\
\hline \multirow[t]{3}{*}{13} & Siempre $(9,6 \%)$ \\
\hline & Nunca $(34,9 \%)$ \\
\hline & $\begin{array}{l}\text { Siempre que tenga antecedentes } \\
\text { de cardiopatía }(55,4 \%)\end{array}$ \\
\hline \multirow[t]{3}{*}{14} & $70 \mathrm{~g} / \mathrm{l}(67,9 \%)$ \\
\hline & $90 \mathrm{~g} / \mathrm{l}(10,3 \%)$ \\
\hline & $\begin{array}{l}\text { No indicaría transfusión, } \\
\text { independientemente de la hemoglobina } \\
\text { (Hb) }(21,8 \%)\end{array}$ \\
\hline \multirow[t]{3}{*}{15} & $70 \mathrm{~g} / 1(72,2 \%)$ \\
\hline & $90 \mathrm{~g} / 1(20,3 \%)$ \\
\hline & $\begin{array}{l}\text { No indicaría transfusión, } \\
\text { independientemente de la } \mathrm{Hb}(7,6 \%)\end{array}$ \\
\hline \multirow[t]{3}{*}{16} & $70 \mathrm{~g} / \mathrm{l}(72,3 \%)$ \\
\hline & $90 \mathrm{~g} / 1(9,6 \%)$ \\
\hline & $\begin{array}{l}\text { No indicaría transfusión, } \\
\text { independientemente de la } \mathrm{Hb}(18,1 \%)\end{array}$ \\
\hline \multirow[t]{3}{*}{17} & $70 \mathrm{~g} / \mathrm{l}(60,5 \%)$ \\
\hline & $90 \mathrm{~g} / 1(32,9 \%)$ \\
\hline & $\begin{array}{l}\text { No indicaría transfusión, } \\
\text { independientemente de la } \mathrm{Hb}(6,6 \%)\end{array}$ \\
\hline \multirow[t]{6}{*}{18} & Plaquetas posquirúrgicas $<30 / 10^{9}(61,9 \%)$ \\
\hline & $\begin{array}{l}\text { Plaquetas posquirúrgicas }<30 / 10^{9}+ \\
\text { antiagregantes }(21,4 \%)\end{array}$ \\
\hline & Plaquetas posquirúrgicas $<100 / 10^{9}(9,5 \%)$ \\
\hline & $\begin{array}{l}\text { Transfusión }>10 \text { unidades de concentrado } \\
\text { de hematíes }(2,4 \%)\end{array}$ \\
\hline & $\begin{array}{l}\text { Toma antiagregantes plaquetarios en la } \\
\text { semana previa }(2,4 \%)\end{array}$ \\
\hline & $\begin{array}{l}\text { Plaquetas posquirúrgicas }<30 / 10^{9}+ \\
\text { concentrados de hematíes }(2,4 \%)\end{array}$ \\
\hline \multirow[t]{2}{*}{19} & $\begin{array}{l}\text { Sí, la } \mathrm{Hb} \text { es un factor más en la decisión } \\
\text { de transfundir }(88,1 \%)\end{array}$ \\
\hline & $\begin{array}{l}\text { No, el valor de hemoglobina es el marcador } \\
\text { fundamental por el que decido transfundir } \\
(11,9 \%)\end{array}$ \\
\hline \multirow[t]{3}{*}{20} & $\begin{array}{l}\text { Se prescriben acorde al estudio de } \\
\text { coagulación }(63,1 \%)\end{array}$ \\
\hline & $\begin{array}{l}\text { Se prescriben sólo en situaciones muy } \\
\text { especiales y si no hay alternativas } \\
\text { farmacológicas }(32,1 \%)\end{array}$ \\
\hline & $\begin{array}{l}\text { Se prescriben acorde al estudio de } \\
\text { coagulación }(4,8 \%)\end{array}$ \\
\hline \multirow[t]{3}{*}{26} & $\begin{array}{l}\text { Sí, clara asociación; las transfusiones causan } \\
\text { aumento de la morbilidad }(44 \%)\end{array}$ \\
\hline & $\begin{array}{l}\text { Sí, asociación circunstancial entre } \\
\text { transfusiones y aumento de la } \\
\text { morbimortalidad }(34,5 \%)\end{array}$ \\
\hline & No conozco los estudios referidos $(21,4 \%)$ \\
\hline
\end{tabular}

ner una visión general de las actitudes y conductas. La distribución geográfica de las unidades abarca toda la extensión nacional, con mayor número de unidades en la Comunidad de Madrid (15 unidades), seguido por Cataluña (14 unidades), Comunidad Valenciana, Castilla-La Mancha y Castilla y León (8 unidades cada una de las tres últimas). Hay un número similar de hospitales de nivel I-II y hospitales de nivel III, con una media de 10 a 20 camas en la UCI con una actividad predominantemente médica. No hay ninguna UCI monotemática, aunque los enfermos politraumatizados son tratados en la mayoría de ellas.
En la encuesta el adjunto responsable toma la decisión de transfundir en la mayoría de las ocasiones, según su experiencia, pero con tendencia a seguir las recomendaciones científicas. A esto ayuda la falta de comités de transfusiones o su poca representatividad práctica en la mayoría de los centros.

Cuando se analiza el porcentaje de pacientes transfundidos, observamos que el 15-25\% de los pacientes reciben plasma fresco congelado, y menos del $5 \%$, concentrados de plaquetas. Probablemente, dada la limitación de los cuestionarios para valorar estos datos, los resultados están sobredimensionados (poca validez), pero presentan una gran fiabilidad (resultados constantes). Los pacientes reciben un aporte habitual de 2-4 concentrados de hematíes, cifras que pueden ser adecuadas en contextos de sangrado agudo, pero excesivas en pacientes críticos con anemia no hemorrágica. Así y todo la tasa de pacientes en los que se transfunde sólo una unidad de concentrados de hematíes es sólo del 14,3\%.

Hemos establecido unas preguntas sobre un contexto clínico de transfusión que permite ver la actuación en determinadas circunstancias. Aunque la cifra de hemoglobina es sólo orientativa en la toma de decisiones, la indicación es clara cuando es $<7 \mathrm{~g} / \mathrm{dl}$ (< $10 \mathrm{~g} / \mathrm{dl}$ en pacientes cardiópatas). Ha calado la política de indicación más estricta de hemoderivados, con cifras similares a las consensuadas ${ }^{5}$.

Se sigue sin confiar en las diferentes alternativas farmacológicas a las transfusiones, fundamentalmente por su escasa evidencia y su precio. Es destacable la escasa presencia de dispositivos recuperadores de sangre, con utilización esporádica de aprotinina, eritropoyetina ( $<5$ casos/año) y factor VII activado. Es posible que estos datos hayan cambiado, fundamentalmente hacia su aumento, en los últimos años ${ }^{20-26}$.

Sólo en menos de la mitad de las encuestas se considera clara la relación entre la transfusión y la mortalidad. Creemos necesario remarcar este concepto, bastante probado en la actualidad (y en el momento del estudio), que obligaría al clínico a pensar en continuar con medidas de ahorro de hemoderivados y en buscar alternativas a su utilización, así como evaluar de forma continua sus efectos deletéreos en supervivencia y complicaciones ${ }^{6,7}$.

Como conclusión podemos decir que debemos seguir intentando reducir la transfusión en los enfermos críticos y evitar la sobretransfusión, pues no solamente es un elemento deficitario, sino que tiene consecuencias deletéreas en la evolución del paciente. Esto nos hará seguir buscando y utilizando las alternativas farmacológicas que nos sirvan de apoyo ${ }^{27}$. Por otra parte, nunca se dejará de insistir en que la política transfusional no es un acto aislado de cada UCI, sino que se necesitan protocolos educacionales, conferencias de consenso y una mayor participación de los diferentes servicios implicados (en forma de comité de transfusiones).

La repetición de encuestas similares nos irá dando una idea de cómo evoluciona la forma práctica que tiene el médico intensivista de afrontar la transfusión. 


\section{BIBLIOGRAFÍA}

1. Hajjar LA, Auler Junior JO, Santos L, Galas F. Blood transfusión in critically ill patients: state of the art. Clinics. 2007;62: 507-24.

2. Muñoz M, Leal-Noval SR, García-Erce JA, Naveira E. Prevalencia y tratamiento de la anemia en el paciente crítico. Med Intensiva. 2007;31:388-98.

3. Lauzier F, Cook D, Griffith L, Upton J, Crowther M. Fresh frozen plasma transfusion in critically ill patients. Crit Care Med. 2007;35:1655-9.

4. Salman SS, Fernández Pérez ER, Stubbs JR, Gajic O. The practice of platelet transfusión in the intensive care unit. J Intensive Care Med. 2007;22:105-10.

5. Dellinger RP, Levy MM, Carlet JM, Bion J, Parker MM, Jaeschke R, et al. Surviving sepsis campaign: international guidelines for management of severe sepsis and septic shock: 2008. Crit Care Med. 2008;36:296-327.

6. Vincent JL, Baron JF, Reinhart K, Gattinoni L, Thijs L, Webb A, et al. Anemia and blood transfusion in critically ill patients. JAMA. 2002;288:1499-507.

7. Corwin HL, Gettinger A, Pearl RG, Fink MP, Levy MM, Abraham E, et al. The CRIT Study: anemia and blood transfusion in the critically ill-current clinical practice in the United State. Crit Care Med. 2004;32:39-52.

8. Nuttall GA, Stehling LC, Beighley CM, Faust RJ. Current transfusion practices of members of the American Society of Anesthesiologists. Anesthesiology. 2003;99:1433-43

9. Llau Pitarch JV, Sánchez de Merás AM. [Transfusion practice of anesthesiologists in Spain]. Rev Esp Anestesiol Reanim. 1998;45:226-32.

10. Kaufmann J, Klimek M, Kampe S, Paul M Kasper SM. A survey of autologous blood transfusion practises in Germany. Transfus Med. 2004;14:335-41.

11. Wong EC, Perez-Albuerne E, Moscow JA. Transfusion management strategies: a survey of practicing pediatric hematology/ oncology specialist. Pediatr Blood Cancer. 2005;44:119-27.

12. Nahum E, Ben-Ari J, Schonfeld T. Blood Transfusion policy among European pediatric intensive physicians. J Intensive Care Med. 2004;19:38-43.

13. Fung MK, Downes KA, Shulma IA. Transfusion of platelets containing ABO-incompatible plasma: a survey of 3156 North American laboratories. Arch Pathol Lab Med. 2007;31:99-116.

14. McIntyre LA, Hébert PC, Fergusson D, Cook DJ, Aziz A; the Canadian Critical Care Trials Group. A survey of Canadian intensivists'resuscitation practices in early shock. Crit Care. 2007; 11:R74.
15. Kopko P, Silva M, Shulman I, Kleinman S. AABB survey of transfusion-related acute lung injury policies and practices in the United States. Transfusion. 2007;47:1679-85.

16. Brown E, Bennett M. Survey of blood transfusion practice for palliative care patients in Yorkshire: implications for clinica care. J Palliate Med. 2007;10:919-22.

17. Puppo M, Ridolfi A, Sánchez Nuñez G. Evaluación del conocimiento sobre la práctica transfusional entre médicos de tres hospitales públicos de Chaco y Formosa. Rev Argent Transfus. 2001;27:620-5

18. Cabanes F, Sangüesa MJ. Resultados de la encuesta sobre práctica transfusional entre traumatólogos-ortopedas en España. Revista de Ortopedia y Traumatología. 2004;48:449-54

19. Hebert PC, Wells G, Martin C, Tweeddale M, Marshall J, Blajchman M, et al. A Canadian survey of transfusion practices in critically ill patients. Crit Care Med. 1998;26:482-7.

20. Alberca I, Asuero MS, Bóveda JL, Carpio N, Contreras E, Fernández-Mondejar E, et al. Documento Sevilla de Consenso sobre alternativas a la transfusión de sangre alogénica. Med Clin (Barc). 2006;127 Supl 1:3-20.

21. Mayer SA, Brun NC, Bergtrup $K$, Broderick J, Davis $S$, Diringer MN, et al, for the Recombinant Activated Factor VII Intracerebral Hemorrhage Trial Investigators. Recombinant activated factor VII for acute intracerebral hemorrhage. N Engl J Med. 2005;352:777-85

22. Boffard KD, Riou B, Warren B, Chomg PIT, Rizoli S, Rossaint R, et al. Recombinant factor VIIa as adjunctive therapy for bleeding control in severely injured trauma patients: two parallel randomized, placebo-controlled, double-blind clinical trials. Trauma. 2005;59:8-18.

23. Lecumberri R, Páramo JA, Hidalgo F, Feliu J, Iglesias R, Rocha E. Reducción de las necesidades transfusionales en hemorragias adquiridas graves mediante factor VII activo recombinante Med Clin (Barc). 2005; 125:252-3.

24. Quintana M, Sánchez Casado M, García de Lorenzo A. Factor VII activado recombinante. Med Intensiva. 2007;31 326-30.

25. Van der Linden PJ, Harde JF, Daper A, Trenchant A, De Hert SG. Cardiac surgery with cardiopulmonary bypass: does aprotinin affect outcome? Br J Anaesth. 2007;99:646-52.

26. Furnary AP, Wu Y, Hiratzka LF, Grunkemeier GL, Page US 3rd. Aprotinin does not increase the risk of renal failure in cardiac surgery patients. Circulation. 2007;116 Suppl 11:I127-33.

27. Tinmouth A. Reducing the amount of blood transfused by changing clinician, transfusion practices. Transfusion. 2007;47 Suppl 2:S132-6. 